\title{
Shear-enhanced adsorption of a homopolymeric globule mediated by surface catch bonds
}

\author{
Matthias Radtke ${ }^{\mathrm{a}}$ and Roland R. Netz \\ Fachbereich Physik, Freie Universität Berlin, Arnimallee 14, 14195 Berlin, Germany
}

Received 7 March 2015 and Received in final form 19 May 2015

Published online: 30 June 2015

(c) The Author(s) 2015. This article is published with open access at Springerlink.com

\begin{abstract}
The adsorption of a single collapsed homopolymer onto a planar smooth surface in shear flow is investigated by means of Brownian hydrodynamics simulation. While cohesive intra-polymer forces are modeled by Lennard-Jones potentials, surface-monomer interactions are described by stochastic bonds whose two-state kinetics is characterized by three parameters: bond formation rate, bond dissociation rate and an effective catch bond parameter that describes how the force acting on a surface-monomer bond influences the dissociation rate. We construct adsorption state diagrams as a function of shear rate and all three surface-monomer bond parameters. We find shear-induced adsorption in a small range of parameters for low dissociation and association rates and only when the surface-monomer bond is near the transition between slip and catch bond behavior. By mapping on a simple surface-monomer interaction model with conservative pair potentials we try to estimate the conservative potential parameters necessary to observe shear-induced surface adsorption phenomena.
\end{abstract}

\section{Introduction}

Some biological bonds exhibit prolonged lifetimes in the presence of tensile forces, a counter-intuitive phenomenon referred to as catch bond behavior $[1,2]$. Experimental evidence for catch bond behavior comes from studies on various receptor-ligand complexes [3-7] and motivated atomistic $[8,9]$ as well as mesoscopic [10] simulation studies with the goal to characterize and explain the underlying mechanisms. As a matter of fact, also force-insensitive bonds have been reported [7], which can be viewed as intermediate between catch bonds and the more common slip bonds.

How individual biological bonds respond to forces has profound implications for the surface-adhesion of biomacromolecules under flow conditions. The present study is motivated by the blood protein von Willebrand Factor (VWF), which plays a key role in hemostasis [11] by unfolding and activation at elevated shear rates and subsequent binding to the vessel wall [11-13]. Catch bond behavior has been found in the binding of VWF A1 domain to platelets' GPIb $\alpha[4,6]$, and also the collagen-mediated binding of VWF to blood vessel walls was suggested to exhibit a non-trivial force dependence [12].

Recently, we have shown that for simple coarse-grained polymer models based on a time-independent Hamiltonian entirely consisting of energy-conserving pair poten-

\footnotetext{
a e-mail: radtkem@zedat.fu-berlin.de
}

tials between monomers and the surface, adsorption is not enhanced in the presence of shear flow [14], i.e., hydrodynamic shear always favors the desorbed state of a single globular or coiled polymer $[15,16]$. This stands in contrast to experimental findings on VWF adhesion under high flow conditions [12], and thus suggests that in order to obtain shear-induced adsorption behavior, slip-resistant catch bonds might be a necessary ingredient. That catch bonds are in fact sufficient to induce adsorption of polymeric globules on surfaces by shear has been demonstrated in the pioneering work by Sing and AlexanderKatz $[17,18]$, an observation that forms the starting point for the present investigations.

Here, we study the adsorption of globular polymers by stochastic two-state surface-monomer bonds, which is a model that for particular parameter values was previously shown to lead to shear-induced adsorption [17]. Similar models with simple two-state kinetics have proven useful in a number of studies since it is relatively straightforward to include the force-dependent bond stability in a heuristic manner so that either slip or catch bond behavior is obtained [17-21]

We present adsorption state diagrams as a function of shear rate, the surface-monomer bond dissociation and association rates and an effective catch bond parameter that describes the continuous change from slip to catch bond behavior. The adsorption transition displays shearinduced adsorption only for rather low dissociation and 
association rate and only for bonds that show neither pronounced slip nor catch bond behavior.

In the last part, we compare the stochastic two-state surface-monomer bond model with a binding scenario based on conservative surface-monomer potentials, similar to that used in our previous study [14]. We find that in order to see shear-induced adsorption phenomena, surfacemonomer potentials should presumably have an extremely short spatial range, which makes simulations impractical for large system sizes.

\section{Simulation method}

Brownian hydrodynamics simulations are performed using the discretized Langevin equation

$$
\begin{aligned}
& \mathbf{r}_{i}(t+\Delta t)-\mathbf{r}_{i}(t)= \\
& \left(\boldsymbol{\mu}_{i i} \dot{\gamma} z_{i} \hat{\mathbf{x}}-\sum_{j=1}^{N} \boldsymbol{\mu}_{i j} \cdot \nabla_{\mathbf{r}_{j}} U(t)+\sum_{j} \nabla_{\mathbf{r}_{j}} \boldsymbol{\mu}_{i j}\right) \Delta t+\boldsymbol{\xi}_{i}(t),
\end{aligned}
$$

which describes the displacement of bead $i$ during a time step $\Delta t$. Note that all quantities are made dimensionless by rescaling lengths by the monomer radius $a$ according to $r=\tilde{r} / a$, energies $U=\tilde{U} / k_{B} T$ by the thermal energy and times $t=\tilde{t} / \tau$ by the characteristic monomer diffusion time $\tau=a^{2} / \mu_{0} k_{B} T=6 \pi \eta a^{3} / k_{B} T$, with Stokes mobility $\mu_{0}$ and viscosity $\eta$. The first term in eq. (1) represents a linear shear flow with rate $\dot{\gamma}=\tilde{\dot{\gamma}} \tau$, where $\hat{\mathbf{x}}$ is the unit vector in the $x$-direction. The second term accounts for the direct force acting on particle $i$ itself as well as the hydrodynamic flow-field created by forces acting on all other particles $j \neq i$. Hydrodynamic interactions at a surface with noslip boundary condition are taken into account via the mobility matrix approximated by the Rotne-Prager-Blake tensor [22-24] given by

$$
\boldsymbol{\mu}_{i j}=\tilde{\boldsymbol{\mu}}_{i j} / \mu_{0}=\boldsymbol{\mu}_{\mathrm{self}}^{\mathrm{RPB}}\left(z_{i}\right) \delta_{i j}+\left(1-\delta_{i j}\right) \boldsymbol{\mu}^{\mathrm{RPB}}\left(\mathbf{r}_{i}, \mathbf{r}_{j}\right) .
$$

Explicit expressions for the self-mobilities $\boldsymbol{\mu}_{\mathrm{self}}^{\mathrm{RPB}}\left(z_{i}\right)$ and the off-diagonal elements $\boldsymbol{\mu}^{\mathrm{RPB}}\left(\mathbf{r}_{i}, \mathbf{r}_{j}\right)$ have been given previously [24]. The third term in eq. (1) compensates for the spurious flux due to inhomogeneities in the selfmobility [25]. The stochastic contribution $\boldsymbol{\xi}_{i}$ is given by Gaussian random vectors with correlations according to the fluctuation-dissipation theorem $\left\langle\boldsymbol{\xi}_{i}(t) \boldsymbol{\xi}_{j}\left(t^{\prime}\right)\right\rangle=$ $6 \boldsymbol{\mu}_{i j} \Delta t \delta\left(t-t^{\prime}\right)$ and vanishing mean. The simulations typically run for $10^{9}$ time steps of length $\Delta t=10^{-4}$. For computational speed we update the mobility matrix $\boldsymbol{\mu}_{i j}$ only every 100 time steps, which does not alter the results.

The homopolymer model consists of $N=50$ beads, which interact via Lennard-Jones potentials of depth $\varepsilon=\tilde{\varepsilon} / k_{B} T=2$ and are connected in a linear chain by harmonic bonds with a rescaled spring constant $\kappa=$ $\tilde{\kappa} a^{2} / k_{B} T=200$. The value chosen for $\varepsilon$ corresponds to a strongly collapsed globule, which, however, is still far from a crystallization transition that for $N=50$ occurs at about $\varepsilon=4$ [26]. The intra-polymer potential is given by

$$
U_{\text {pol }}=\varepsilon \sum_{i<j}\left(\left(2 / r_{i j}\right)^{12}-2\left(2 / r_{i j}\right)^{6}\right)+\frac{\kappa}{2} \sum_{i}\left(r_{i, i+1}-2\right)^{2}
$$

The total potential energy $U=U_{\text {pol }}+U_{\text {rep }}$ includes in addition a surface term $U_{\text {rep }}$ that accounts for steric exclusion of the polymer from the surface and in particular prevents crossing of the no-slip boundary located at $z=0$. We use

$$
\begin{aligned}
& U_{\text {rep }}= \\
& \sum_{i} \begin{cases}2 \pi \sigma_{R}^{2}\left(\frac{2}{5}\left(\frac{\sigma_{R}}{z_{i}}\right)^{10}-\left(\frac{\sigma_{R}}{z_{i}}\right)^{4}+\frac{3}{5}\right), & z_{i} \leq \sigma_{R}, \\
0, & z_{i}>\sigma_{R},\end{cases}
\end{aligned}
$$

with a short range of $\sigma_{R}=1.2$. The maximal height above the surface is restricted by a soft wall acting on the polymer center-of-mass located at a height $z=15$.

Surface adhesion is modeled via surface-monomer bonds that are governed by stochastic two-state kinetics. A monomer can reversibly bind to the surface when it is within the surface reaction range $z_{r}=2$ above the no-slip boundary, regardless of its lateral position. The binding probability is determined by the adsorption rate. When the monomer is bound its mobility is set to zero, i.e., the position is frozen despite hydrodynamic and direct forces due to other beads. The virtual force acting on an immobilized monomer is not included in the off-diagonal mobility terms in eq. (1), which means that an immobilized monomer is transparent to the flow or, in other words, that it perfectly fits into surface defects without modifying the surface flow boundary condition. Adsorption and desorption rates can be interpreted in terms of energy barrier heights $E_{a}$ and $E_{d}$, respectively, in a fictitious energy landscape. The equilibrium behavior is governed by the energy difference $\Delta E=E_{d}-E_{a}$. With a pre-factor $\nu$, the so-called attempt frequency, the adsorption rate becomes

$$
k_{\mathrm{a}}=\nu e^{-E_{a}},
$$

while the desorption rate is given by

$$
k_{\mathrm{d}}=\nu e^{-\left(E_{d}-x f\right)},
$$

where the catch bond parameter $x$ sets the sensitivity of the desorption rate to the force $f$ acting on the bound particle $i$, defined by $f=\left|\dot{\gamma} z_{i} \hat{\mathbf{x}}-\nabla_{\mathbf{r}_{i}} U\right|$. In the calculation of $f$ we omit stochastic forces, which have vanishing mean, as well as hydrodynamic interactions, considering immobilized monomers to be transparent to the flow field created by other monomers, as discussed earlier. Attempts for bond formation for each monomer within the surface reaction range, $z \leq z_{r}=2$, and dissociation for each existing surface bond are performed with a frequency $\nu=\tilde{\nu} \tau=100$. Thus, in the simulation, bonds are updated every 100 time steps $\left(\Delta t=10^{-4}\right)$ and the bond force $f$ is 
a)

b)

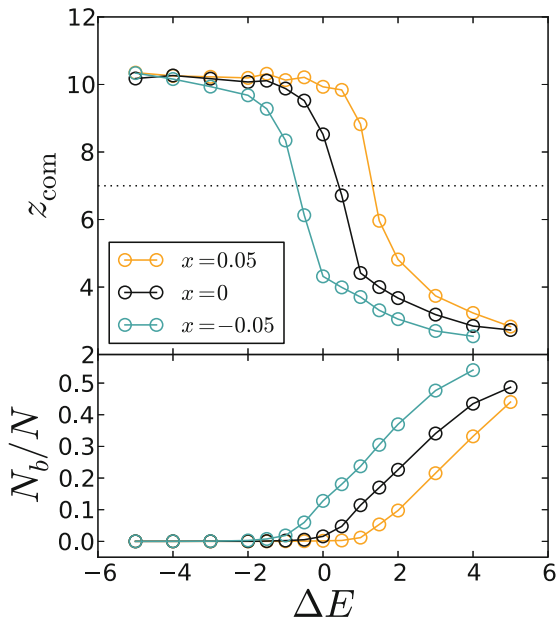

c)

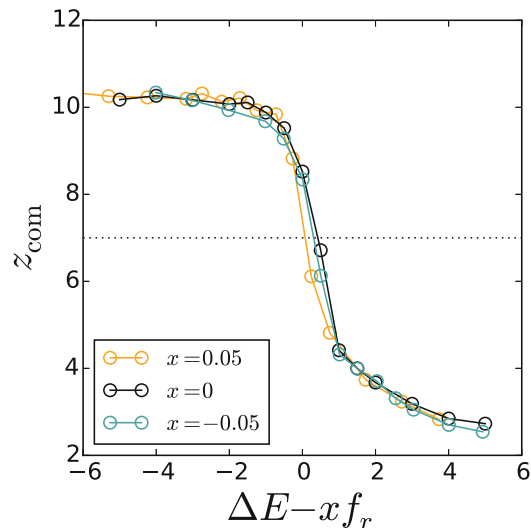

d)

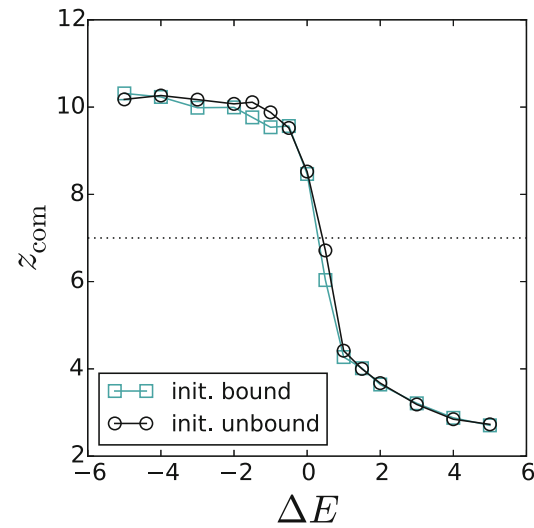

Fig. 1. Adsorption of a collapsed polymer with cohesive strength $\varepsilon=2$ and number of monomers $N=50$ in the absence of shear flow. The adsorption energy barrier is fixed at $E_{a}=9$. A horizontal black dotted line indicates the adsorption transition threshold defined as $z_{\text {com }}=7$. a) The average height of the polymer center-of-mass $z_{\text {com }}$ and b) the rescaled average number of bonds $N_{b} / N$ are plotted as a function of the adsorption energy $\Delta E$. Upon changing from slip bond behavior, $x=0.05$ (orange), to catch bonds, $x=-0.05$ (cyan), the equilibrium adsorption transition shifts towards smaller $\Delta E$. c) When the adsorption energy is shifted by the average force acting on the surface bonds at the moment of rupture, $\Delta E-x f_{r}$, the data for $z_{\text {com fall on }}$ a single curve regardless of the value of $x$. d) The equilibrium adsorption behavior is independent of the initial configuration, as demonstrated for $x=0$; simulations are either initialized in the desorbed state (black) or in a state where the globule is bound to the surface via a randomly chosen bead (cyan).

averaged during this time interval. Note that the update of the mobility matrix $\boldsymbol{\mu}_{i j}$ occurs with the same frequency. As opposed to a similar study [17] we do not represent surface bonds by harmonic springs which are suddenly switched on, as this can lead to abrupt changes of tensile spring forces for newly formed bonds and thereby affect the balance between dissociation and association rates.

\section{Results}

\subsection{Equilibrium adsorption}

First we consider the adsorption behavior of a collapsed polymer in equilibrium without shear flow. As shown in fig. 1a), the average height of the center-of-mass $z_{\mathrm{com}}=$ $N^{-1} \sum_{i}^{N} z_{i}$ relative to the surface decreases with rising adsorption energy $\Delta E$ while the rescaled average number of bonds $N_{b} / N$ in fig. 1b) increases. The adsorption transition, indicated by the dotted horizontal line, is defined by the distance criterion $z_{\text {com }}=7$, which is chosen so that $z_{\text {com }}$ exhibits maximal slope at the adsorption transition, as illustrated in fig 1a). The average number of bonds at the adsorption transition is of the order of $N_{b} / N \approx 0.05$, meaning that roughly two to three surface-monomer bonds are present at the transition. Upon changing from slip bond, $x>0$, to catch bond behavior, $x \leq 0$, the equilibrium adsorption transition shifts towards smaller values of $\Delta E$, meaning that catch bond behavior enhances the adsorption. This follows from the fact that the ratio of the adsorption and desorption rates defined in eqs. (5), (6) is given by $k_{a} / k_{d}=e^{\Delta E-x f}$ where the force $f$ acting on the surface bond is a positive definite quantity. As shown in fig. 1c), the average height $z_{\text {com }}$ for different values of $x$ fall on a single curve when plotted as a function of $\Delta E-x f_{r}$, where $f_{r}$ is the force acting on the bond at the moment of rupture. In fig. 1d) we confirm that the simulation results do not depend on the initial conditions, i.e. there is no difference in the equilibrium globule height for simulations initialized with a desorbed, unbound globule and simulations initialized with an adsorbed globule where a single, randomly chosen bead is bound to the surface. For the results shown in fig. 1, the force-independent adsorption energy barrier is kept constant at a value $E_{a}=9$.

\subsection{Varying catch bond parameter at fixed adsorption and desorption rates}

An important question concerns the effect of the catch bond parameter $x$ on the non-equilibrium adsorption behavior in shear flow. For positive values of $x$, the surface bonds exhibit the usual slip behavior and are weakened when a force acts on the bond. Changing the catch bond parameter towards negative values leads to bonds that become stronger under force, which effectively might enhance adsorption in shear flow. Indeed, it has been shown that shear induces adsorption for low desorption rates $k_{\mathrm{d}}$ and for vanishing catch bond parameter $x=0$ [17]. In the following we determine the range of parameters for which adsorption is enhanced by shear.

In order to determine the adsorption transition we plot the average height of the polymer center-of-mass $z_{\text {com }}$ in fig. 2a) as a function of shear rate $\dot{\gamma}$ for a few fixed values 
a)

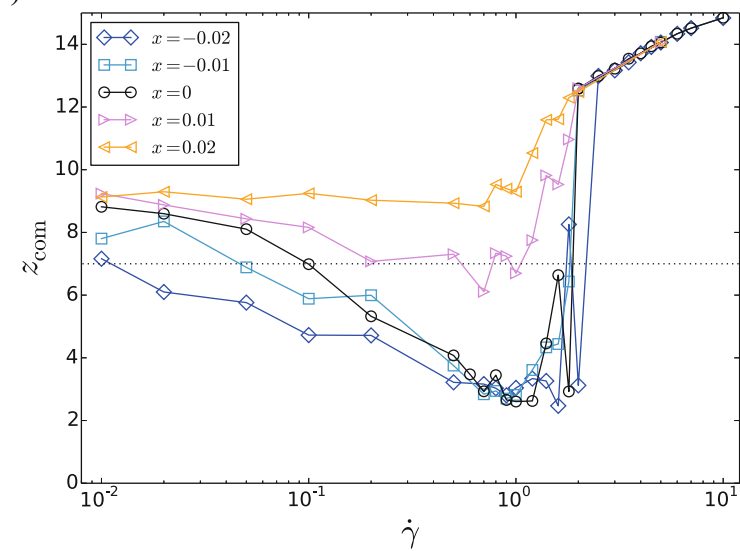

b)

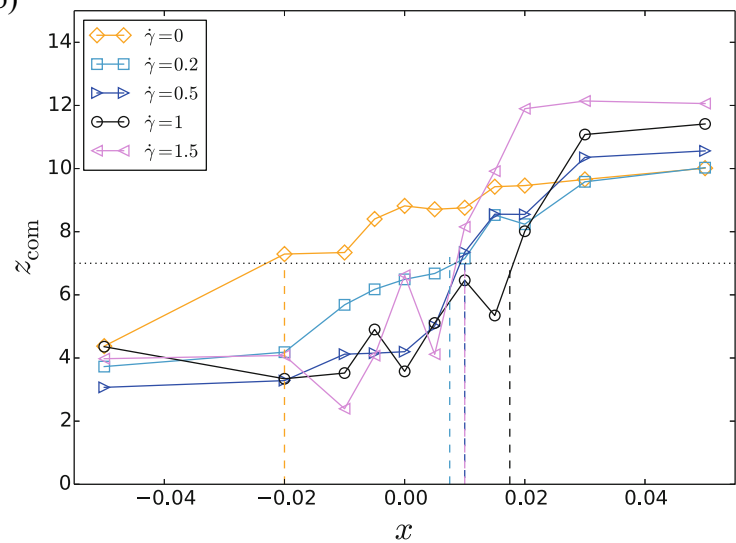

Fig. 2. a) Average height of the polymer center-of-mass $z_{\text {com }}$ as a function of shear rate $\dot{\gamma}$ for different fixed values of the catch bond parameter $x$. Surface bonds are characterized by high adsorption and desorption energy barriers $E_{a}=E_{d}=9$. Whereas $x<0$ corresponds to catch bonds, positive $x$ corresponds to slip bond behavior. The dotted horizontal line indicates the adsorption transition defined by the distance criterion $z_{\mathrm{com}}=7 \mathrm{~b}$ ) Average height $z_{\mathrm{com}}$ as a function of $x$ for different shear rates and fixed $E_{a}=E_{d}=9$. The adsorption transition is indicated by vertical dashed lines.

of the catch bond parameter $x$ and in fig. 2b) as a function of $x$ for a few different fixed values of $\dot{\gamma}$. Low rates of bond formation and dissociation are obtained by choosing high values for the adsorption and desorption energy barriers, $E_{a}=E_{d}=9$. The choice of $\Delta E=E_{d}-E_{a}=0$ ensures proximity to the adsorption transition, as can be seen in fig. 1a)

Indeed, pronounced shear-induced adsorption is observed for $x=0$ in fig. 2a), in agreement with literature results [17]; at low shear rates $\dot{\gamma}<0.1$ the globule is in the desorbed state, defined by the distance criterion $z_{\text {com }}>7$ and indicated by the horizontal dotted line. Further increasing the shear rate leads to adsorption until the desorbing lift force overwhelms the surface adhesion around $\dot{\gamma}=1.9$. Whereas slip bonds with $x=0.02$ always lead to desorption (orange symbols), small negative values of $x$, i.e. catch bond behavior, favors adsorption at low shear

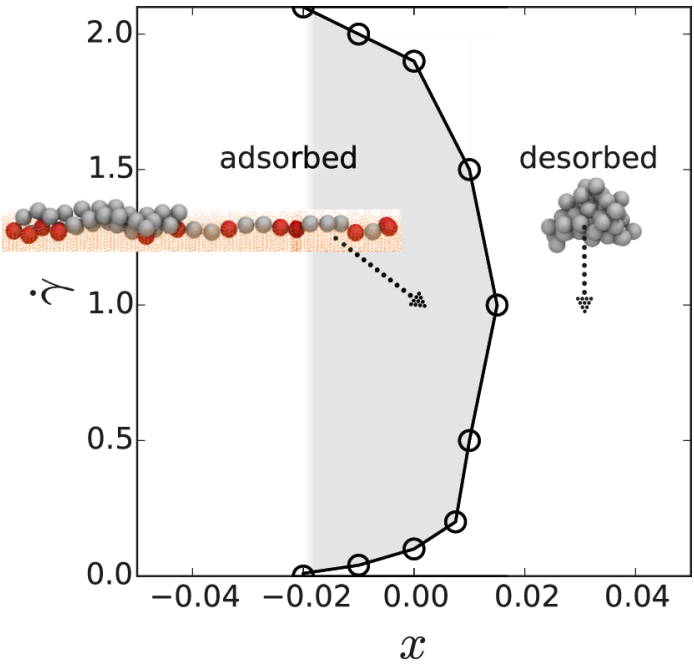

Fig. 3. Adsorption state diagram as a function of shear rate $\dot{\gamma}$ and catch bond parameter $x$ for a collapsed polymer with cohesive strength $\varepsilon=2$. Stochastic surface bonds are characterized by adsorption and desorption energy barriers $E_{a}=E_{d}=9$. Only within the small shaded region $-0.02<x<0.02$, shearinduced adsorption is observed in a finite window of intermediate shear rates. Snapshots illustrate an adsorbed configuration obtained for $\dot{\gamma}=1, x=0$, where beads that are bound to the surface are colored in red, and a desorbed globule obtained for $\dot{\gamma}=1, x=0.03$ in the desorbed part of the state diagram.

rates but with increasing shear the globule also desorbs. As can be seen in fig. 2b), increasing the catch bond parameter $x$ coming from negative values, where the polymer is adsorbed, leads to a desorption transition. This sheardependent desorption transition, induced by changing the bonds from catch to slip behavior, is indicated by vertical dashed lines.

As a result, we obtain the adsorption state diagram in fig. 3, showing the adsorption transition of a collapsed globule as a function of rescaled shear rate $\dot{\gamma}$ and the catch bond parameter $x$. Although the hydrodynamic interactions included in the simulations lead to hydrodynamic lift forces that work against adsorption $[15,16]$, in a small parameter range of $-0.02<x<0.02$ we observe shearinduced adsorption. The state diagram in fig. 3 displays reentrant behavior, i.e., adsorption is only observed within a finite small window of intermediate shear rates. Surprisingly, the existence of shear-induced adsorption is very sensitive to the value of the catch bond parameter $x$. We note that whether the polymer is desorbed at high shear rates depends on the initial conditions of the simulation, as discussed below.

To obtain a feeling for the strong influence of the catch bond parameter on the desorption rate, we present in fig. 4 data for the average rupture force $f_{r}$, defined as the force acting on a bond at the moment of dissociation, for the same parameter values already shown in fig. 2a). According to the adsorption state diagram fig. 3, shear-induced adsorption occurs in the range $-0.02<x<0.02$. In the range of shear rates where the globule is adsorbed, the rupture force in fig. 4 is approximately $f_{r}=22 \pm 1$. According 


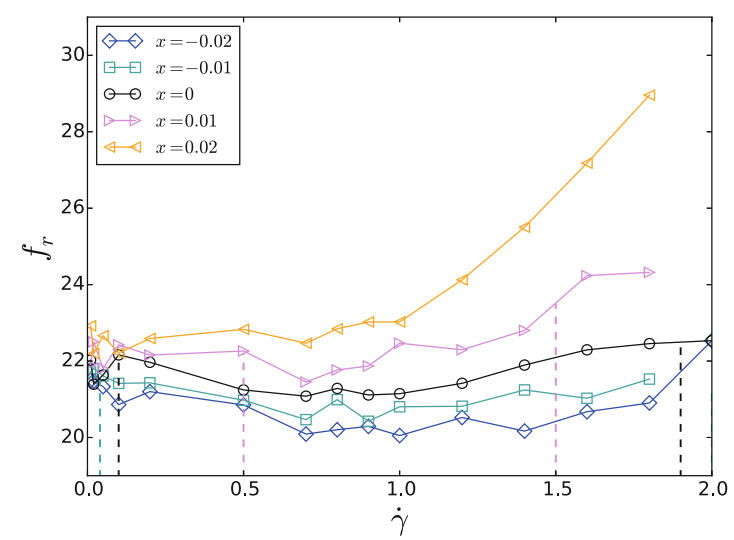

Fig. 4. Average rupture force $f_{r}$ as a function of shear rate $\dot{\gamma}$ for the same parameters as in fig. 2a). Vertical dashed lines indicate the adsorption transition.

to the definition of the desorption rate in eq. (6), the force changes the desorption rate by a factor $e^{x f_{r}}$, which using $f_{r}=22$ translates into $0.64<e^{x f_{r}}<1.55$ for a range of $-0.02<x<0.02$. We conclude that although the catch bond parameter $x$ is very small in the range where shearinduced adsorption is observed, due to the large values of the rupture forces $f_{r}$ the effect on the dissociation rates is sizable, explaining the surprising sensitivity of the resulting adsorption behavior on $x$.

\subsection{Varying adsorption and desorption rates at fixed catch bond parameter}

The shear-dependent adsorption behavior depends on both the adsorption energy barrier $E_{a}$ and the desorption energy barrier $E_{d}$. In fig. 5a) we present the globule center-of-mass height $z_{\text {com }}$ as a function of shear rate for fixed catch bond parameter $x=0$ and fixed adsorption energy barrier height $E_{a}=9$ for a few different values of the adsorption energy $\Delta E=E_{d}-E_{a}$. The data exhibit clear shear-induced adsorption for $\Delta E=0$ (black data points) whereas a positive value $\Delta E=0.5$ leads to adsorption for low shear rates and a negative value $\Delta E=-0.5$ promotes desorption for all values of $\dot{\gamma}$. The effect of varying $\Delta E$ at fixed $x=0$ and $E_{a}=9$ is summarized in the adsorption state diagram fig. 6a). We see that shear-induced adsorption is only observed within a very narrow range of adsorption energies $-0.3<\Delta E<0.4$.

Next we determine the globule adsorption behavior for varying adsorption energy barrier height $E_{a}$ at fixed catch bond parameter $x=0$. For this we fix the adsorption energy at $\Delta E=E_{d}-E_{a}=0$, meaning that we vary both adsorption and desorption barrier heights so that the equilibrium adsorption behavior stays invariant. In fig. 5b) we show the average polymer height as a function of $E_{a}$ for a few fixed shear rates $\dot{\gamma}$ and for $\Delta E=0$, we observe adsorption only for intermediate values of $E_{a}$. These results are summarized in the adsorption state diagram in fig. 6b) as a function of $\dot{\gamma}$ and $E_{a}$. For a whole range of adsorption a)

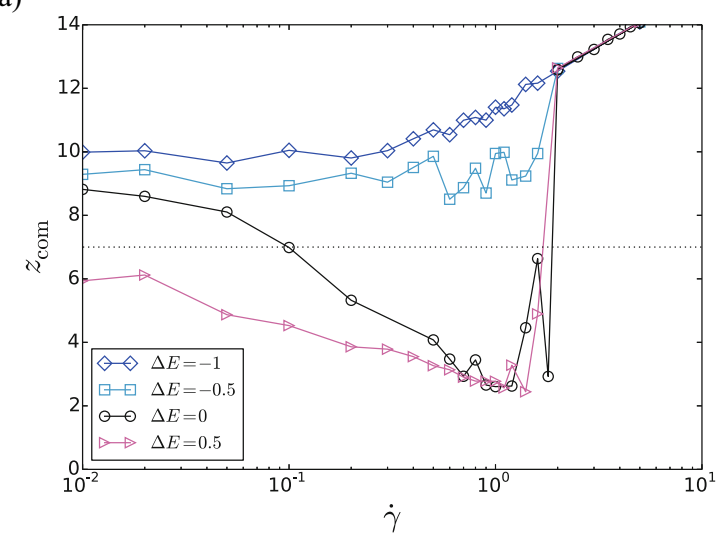

b)

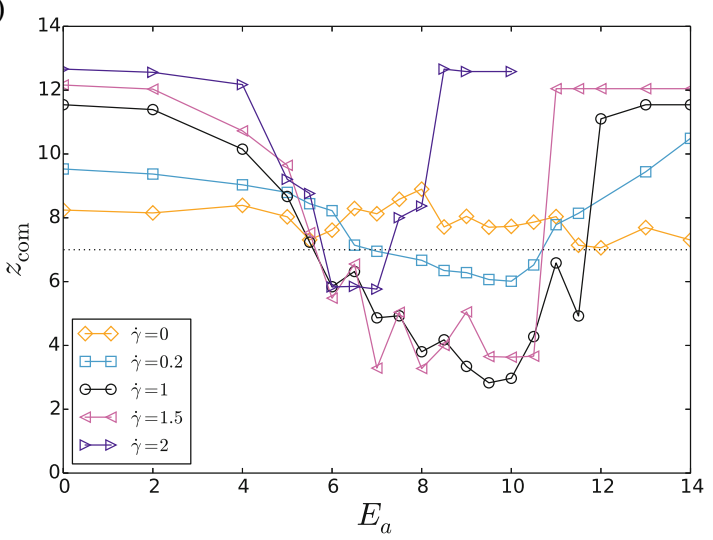

Fig. 5. a) Average height of the polymer center-of-mass $z_{\text {com }}$ as a function of shear rate $\dot{\gamma}$ for a few different values of the adsorption energy $\Delta E=E_{d}-E_{a}$ with fixed $E_{a}=9$ and $x=0$. Whereas negative values of $\Delta E$ always lead to desorption, for $\Delta E>0$ the polymer is adsorbed at low shear flow and with increasing shear rate it crosses the desorption transition defined by $z_{\text {com }}=7$, indicated by the dotted horizontal line. Shearinduced adsorption is observed for $\Delta E=0$. b) Average height $z_{\text {com }}$ as a function of the adsorption energy barrier height $E_{a}$ for fixed $\Delta E=0, x=0$ and a few different shear rates.

energy barrier heights $5<E_{a}<12$ shear-enhanced adsorption is observed for an interval of intermediate shear rates of about $0.1<\dot{\gamma}<2$. As seen in fig. 5b), without shear the polymer is desorbed for all values of $E_{a}$. The adsorption transition is denoted in fig. 6 as black lines. The region shaded in gray indicates where the globule adsorption state depends on the initial conditions of the simulation; at large shear rate and high adsorption energy barrier an initially bound polymer does not desorb during the time of simulation while an initially unbound polymer will stay unbound.

\subsection{Initial condition and reversibility}

The effect of initial conditions and the reversibility of shear-induced adsorption effects are illustrated in fig. 7 . The average height of the polymer above the surface is shown as a function of the adsorption energy barrier height 
a)
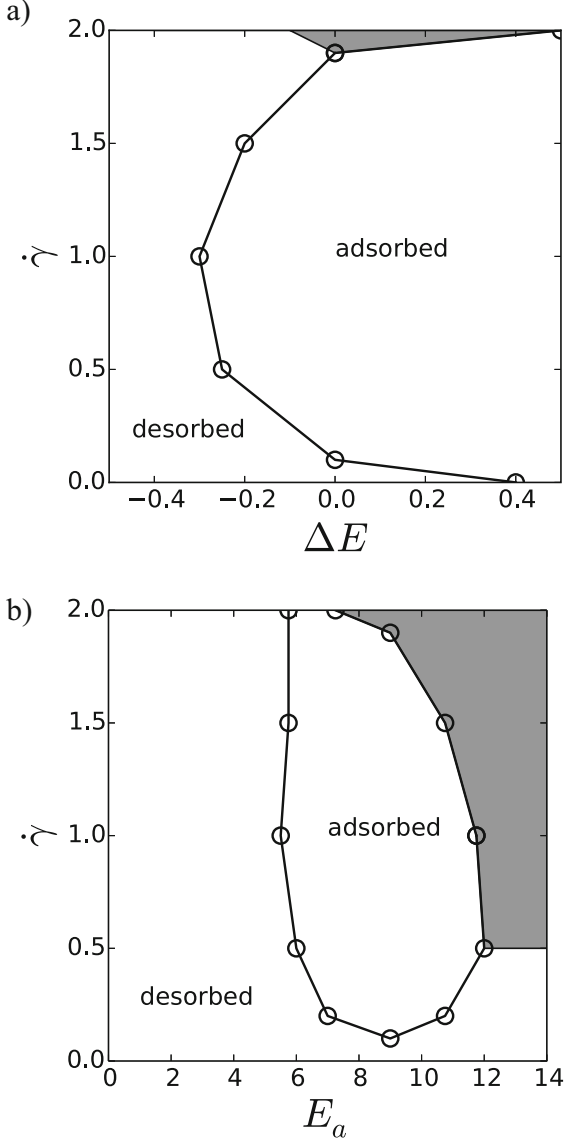

Fig. 6. a) Adsorption state diagram as a function of shear rate $\dot{\gamma}$ and adsorption energy $\Delta E$ at constant adsorption energy barrier height $E_{a}=9$ and catch bond parameter $x=0$. b) Adsorption state diagram as a function of shear rate $\dot{\gamma}$ and adsorption energy barrier height $E_{a}$ for fixed $\Delta E=0$ and $x=0$. In the range $5<E_{a}<12$, shear-induced adsorption is observed for intermediate shear rates. The region shaded in gray, at large $E_{a}$ and large $\dot{\gamma}$, roughly indicates where the adsorption state depends on the initial conditions. Here, during the course of simulations an initially desorbed globule does not adsorb while an initially adsorbed globule remains adsorbed.

$E_{a}$ for fixed $\dot{\gamma}=1, x=0, \Delta E=0$ in fig. 7a), and as a function of shear rate for fixed $E_{a}=9, x=0, \Delta E=0$ in fig. 7b). We compare two different initialization protocols, for the black data points we start each simulation with a desorbed, unbound globule configuration (which is our general protocol used for most simulations), whereas for the cyan data points we start each simulation with a globule that is bound to the surface via a single, randomly chosen bead. Whereas in fig. 7a) the adsorption behavior is independent of the initial condition for $E_{a}<12$, we see that for larger adsorption energy barriers the behavior is determined by the initial conditions. Likewise, for $\dot{\gamma}<2$ in fig. 7b) both simulation protocols lead to identical behavior while for larger shear rate an initially adsorbed globule stays adsorbed and an initially desorbed globule stays desorbed during the course of the simulations which a)

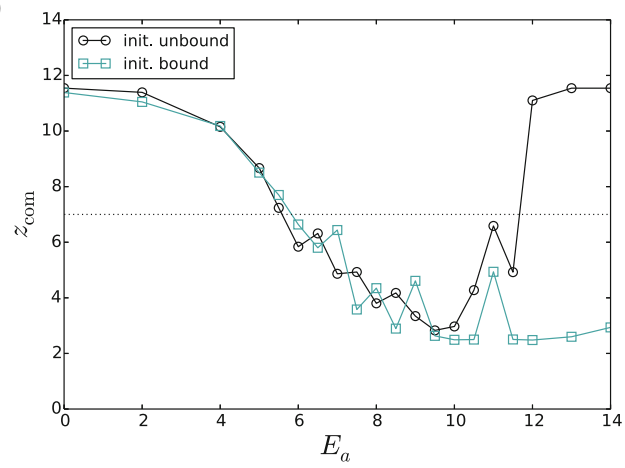

b)

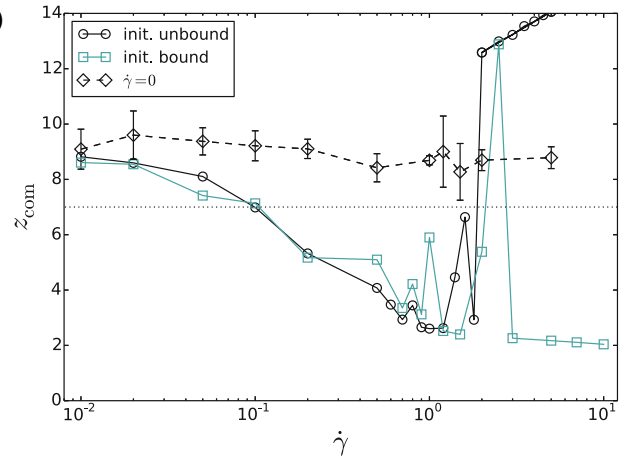

c)

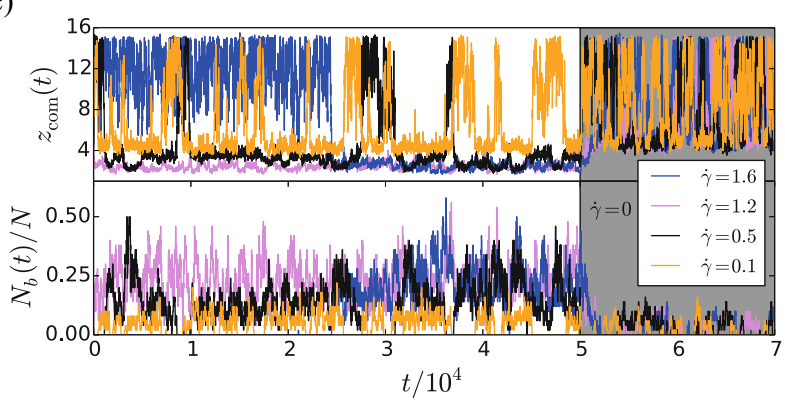

Fig. 7. Effect of different initial conditions and reversibility of the shear-induced adsorption state for fixed parameters $x=0$, $\Delta E=0$. a) Average height $z_{\text {com }}$ as a function of the adsorption barrier $E_{a}$ at fixed $\dot{\gamma}=1$. Simulations that start with a desorbed globule (black data points) exhibit shear-induced adsorption for $5<E_{a}<12$. When the globule is initially adsorbed to the surface (cyan data points) and $E_{a}$ is large, desorption is not observed during the simulation time. b) Similarly, at fixed $E_{a}=9$ and varying shear rate $\dot{\gamma}$, as opposed to simulations starting with a desorbed globule (black solid line), the initially adsorbed globule (cyan) does not desorb at large shear rates. However, the globule reversibly desorbs when the shear flow is suddenly switched off, as indicated by the black dashed line. c) Time series of the instantaneous globule height $z_{\text {com }}(t)$ and the number of surface-monomer bonds $N_{b}(t) / N$ for fixed $E_{a}=9$ and different values of the shear rate $\dot{\gamma}$ as a function of the rescaled simulation time. The initial condition of the simulations is an unbound globule; the globule is adsorbed for all shown shear rates. Within the area shaded in gray, the shear flow is suddenly switched off, $\dot{\gamma}=0$, leading to immediate desorption with a low average number of bonds, indicating reversibility with respect to turning off the shear. 
consist of up to $10^{9}$ simulation steps. We conclude that a large kinetic barrier separates the adsorbed and desorbed states at high shear rate and for high adsorption energy barriers. In the adsorption state diagrams in fig. 6 the region shaded in gray indicates where the adsorption state depends on the initial conditions of the simulation, these are the regions where the system shows irreversible or non-ergodic behavior over the course of the simulations.

The trend towards irreversibility is visualized more explicitly in fig. 7c), where we show time series of the instantaneous height $z_{\text {com }}(t)$ and number of surface bonds $N_{b}(t) / N$ for a few different fixed values of the shear rate. The surface-monomer bond parameters are fixed at $\Delta E=0, E_{a}=9$, and $x=0$ so that the globule exhibits adsorption for shear rates larger than about $\dot{\gamma}=0.1$, as can be gathered from the adsorption state diagrams in fig. 6. Indeed, for a shear rate $\dot{\gamma}=0.1$ (orange line in fig. 7c), which corresponds to the adsorption transition, the globule spends roughly equal amounts of time in the desorbed and in the adsorbed states and frequently switches between the two states. As can be seen in the lower panel of fig. 7c), the adsorbed state is maintained by a rather low average number of surface-monomer bonds of about $N_{b} / N \approx 0.1$. Due to strong hydrodynamic lift forces caused by the shear flow, unbound polymers are most of the time quite far from the surface and outside the adsorption range $z_{r}=2$, preventing surfacemonomer bond formation. Accordingly, for $\dot{\gamma}=1.6$ (blue line in fig. $7 \mathrm{c}$ ), the time span to reach the surface when starting from an unbound state is quite large. As can be seen for the data for $\dot{\gamma}=1.6$, once the globule is adsorbed, more surface-monomer bonds form over time. This cooperative adsorption enhancement in combination with the low dissociation rates, determined by the high desorption energy barrier $E_{d}=9$, makes the desorption of the globule a rare event, which explains why the simulations exhibit irreversible behavior for high shear rates.

On the other hand, the shear-induced adsorbed state shows reversibility with respect to switching off the shear flow. This is demonstrated in fig. 7c), where within the shaded area the flow is turned off and the shear rate set to $\dot{\gamma}=0$. We see that shortly after switching off the shear rate, all trajectories exhibit almost immediate desorption characterized by a low average number of bonds. In fact, this type of reversible behavior is observed for all values of the shear rate, as demonstrated in fig. 7b), where we plot the average globule height after turning off the shear flow (dashed black line).

\section{Mapping between stochastic two-state models and conservative potential models}

As opposed to the stochastic two-state model for surfacemonomer binding used in the present investigation, which exhibits shear-induced globule adsorption for small values of the catch bond parameter $x$, no shear-enhanced adsorption was found in our previous study that employed a description of surface-monomer binding in terms of conservative pair potentials between surface binding sites and monomers [14]. In this section we investigate whether it is possible to find parameters of a conservative surface-monomer potential that might reproduce the shear-induced adsorption behavior seen in the stochastic two-state surface-monomer binding model. To this end, we devise two different ways of extracting the effective parameters $\Delta E, E_{a}$ and $x$ from a conservative potential-based model for surface-monomer interactions.

\subsection{Dissociation rate in a one-dimensional corrugated potential}

First we consider the one-dimensional motion of a single particle, which represents a monomer, that is pulled by an external force $f_{\text {ext }}$ and subject to a corrugated, periodic potential created by a discrete set of Lennard-Jones centers

$$
\begin{aligned}
& U_{\operatorname{inh}}(y)= \\
& \sum_{k} \varepsilon_{W}\left(\frac{\sigma_{W}^{12}}{\left(\sigma_{W}+|y-k b|\right)^{12}}-\frac{2 \sigma_{W}^{6}}{\left(\sigma_{W}+|y-k b|\right)^{6}}\right) .
\end{aligned}
$$

This mimics the potential landscape obtained when decorating a surface with discrete binding sites with distance $b$, interaction range $\sigma_{W}$ and adhesive strength $\varepsilon_{W}$. Figure 8a) displays the resulting potential including the effect of the external pulling force,

$$
U(y)=U_{\mathrm{inh}}(y)-y f_{\mathrm{ext}}
$$

for $b=2$ and two different values of $\sigma_{W}$ and $\varepsilon_{W}$. We study the one-dimensional particle motion using a Brownian Dynamics (BD) simulation scheme. Typical particle trajectories are shown in the inset of fig. 8c) for $b=2$, $\sigma_{W}=0.5, \varepsilon_{W}=10$ and two different values of the external force.

We define the desorption rate $k_{\mathrm{d}}$ by the inverse average bond lifetime, i.e. the time the particle remains within one potential minimum until it crosses the energy barrier and moves to the next binding site. For simplicity we define a bond to be present when the distance between particle and a binding site is smaller than $b / 2$, i.e., the particle is considered always to be bound to the closest surface binding site.

Results from one-dimensional BD simulations in the corrugated potential eq. (8) are shown in fig. 8c) as open symbols, where we plot the desorption rate $k_{\mathrm{d}}$ as a function of the external force $f_{\text {ext }}$ for the two different potential parameters shown in fig. 8a). We remark that the parameter set $\sigma_{W}=0.5$ and $\varepsilon_{W}=10$ (black squares) yields a globule adsorbed in the stick-roll state at a twodimensional inhomogeneous surface [14]. Simulation results are in good agreement with the exact calculation of the escape rate $k_{\mathrm{mfpt}}=1 / \tau_{\mathrm{mfpt}}$ (black line), defined as the inverse mean first passage time of a particle escaping from the minimum of the tilted, corrugated potential eq. (8). 
a)

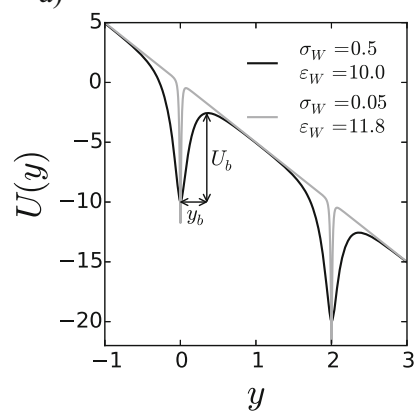

b)
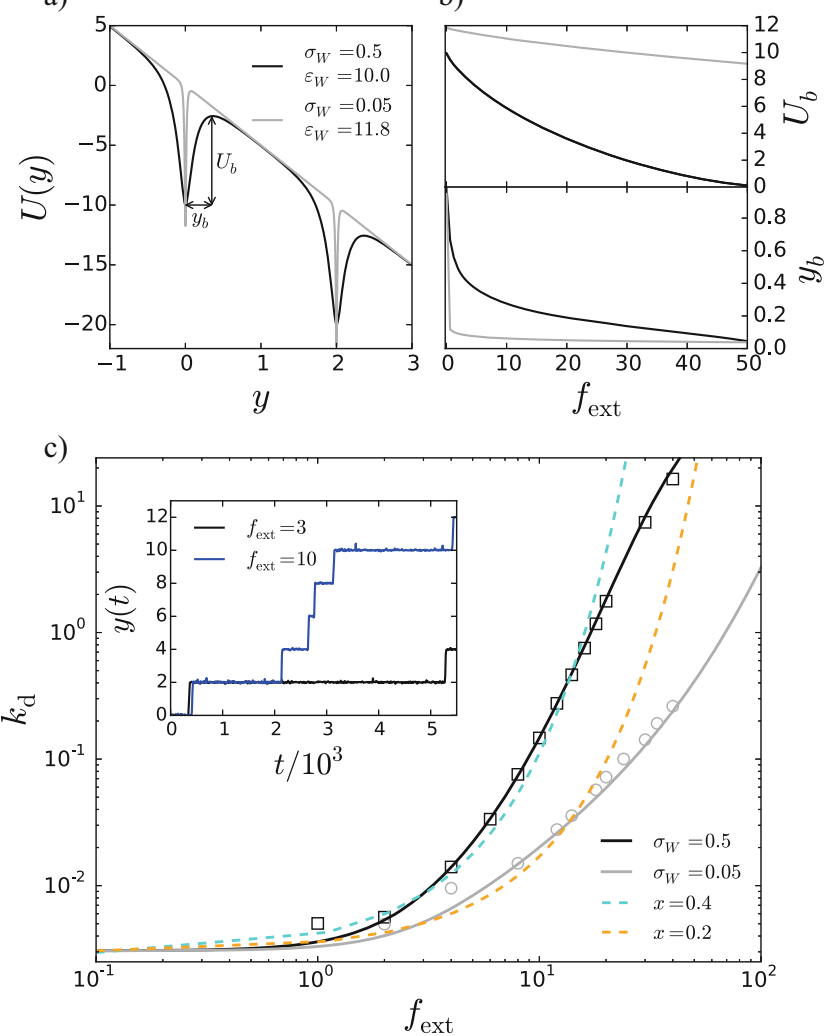

Fig. 8. a) The one-dimensional potential $U(y)$, eq. (8), resulting from the sum over discrete surface binding sites, shown for two different interaction ranges $\sigma_{W}=0.5$ (black) and $\sigma_{W}=0.05$ (gray) with $\varepsilon_{W}=10,11.8$, respectively, and for pulling force $f_{\text {ext }}=5$. b) With increasing force $f_{\text {ext }}$ both the height of the potential energy barrier $U_{b}$ and its distance to the minimum $y_{b}$ decrease. c) Desorption rate $k_{\mathrm{d}}$ as a function of $f_{\text {ext }}$. Results from one-dimensional BD simulations (symbols) are in good agreement with the analytic calculations $k_{\mathrm{mfpt}}$ (solid lines) based on the mean first passage time of a particle escaping from the potential minimum, eq. (9). Exponential fits (colored dashed lines) according to eq. (6) in the range $f_{\text {ext }}<20$ and assuming $\nu=100$ yield desorption energy barriers $E_{d}=10.44,10.42$ and catch bond parameters $x=0.4,0.2$ for $\sigma_{W}=0.5$ and $\sigma_{W}=0.05$, respectively. The inset shows BD simulation trajectories for two different values of $f_{\text {ext }}$ and for $\sigma_{W}=0.5$.

The theoretical result for the mean first passage time [27] to hit either of the two absorbing boundaries at $y= \pm b / 2$, starting in the minimum at $y_{\min }$ is given by

$$
\begin{aligned}
\tau_{\mathrm{mfpt}}= & \frac{\int_{-b / 2}^{b / 2} e^{U(y)} \int_{b / 2}^{y} e^{-U\left(y^{\prime}\right)} \mathrm{d} y \mathrm{~d} y^{\prime}}{\int_{-b / 2}^{b / 2} e^{U(y)} \mathrm{d} y} \int_{-b / 2}^{y_{\min }} e^{U(y)} \mathrm{d} y \\
& -\int_{-b / 2}^{y_{\min }} e^{U(y)} \int_{-b / 2}^{y} e^{-U\left(y^{\prime}\right)} \mathrm{d} y \mathrm{~d} y^{\prime} .
\end{aligned}
$$

For the reduced interaction range $\sigma_{W}=0.05$ (simulation results are plotted as gray circles in fig. 8c), we increase the adhesive strength to $\varepsilon_{W}=11.8$ so that the zero-force desorption rate is the same as for $\sigma_{W}=0.05$ with $\varepsilon_{W}=10$. Here the simulation time step has to be decreased, from $\Delta t=10^{-4}$ to $10^{-7}$, in order to obtain converged results. We see that simulations and the analytical results (gray line) agree very nicely.

In order to extract rate parameters from the model defined by the potential eq. (8), we fit the escape rates according to the desorption rate eq. (6) for forces in the range $f_{\text {ext }}<20$ and using $\nu=100$. We obtain an effective desorption barrier height $E_{d}=10.44$ and a catch bond parameter $x=0.4$ for the potential parameters $\sigma_{W}=0.5$ and $\varepsilon_{W}=10$, the fit is illustrated by the dashed cyan line in fig. 8c). For the more highly corrugated potential, $\sigma_{W}=0.05$ and $\varepsilon_{W}=11.8$, we obtain a smaller catch bond parameter $x=0.2$ but comparable barrier height $E_{d}=10.42$; the fit is illustrated by the orange dashed line in fig. 8c). Note that the fits according to the simple exponential force dependence of the desorption rate in eq. (6) do not describe the actual data in fig. 8c) very well. This can be rationalized by the fact that the actual potential energy barrier height $U_{b}$ and its distance to the minimum $y_{b}$, which are both graphically defined in fig. 8a), depend sensitively on the applied force $f_{\text {ext }}$ and in fact both decrease drastically with increasing $f_{\text {ext }}$, as demonstrated in fig. 8b) for both potentials plotted in fig. 8a). The nonlinear decrease of $U_{b}$ and the force-dependent shift of the barrier height position $y_{b}$ might explain the deviation of the desorption rate from a simple exponential for large forces, which is clearly seen in fig. 8c). In fact, from our fit according to eq. (6) we find apparent barrier heights $E_{d}$ that are comparable to the adhesive strength $\varepsilon_{W}$ in the original potential. By contrast, the fit values for the effective catch bond parameter, which come out as $x=0.4$ and $x=0.2$ for the potential interaction ranges $\sigma_{W}=0.5$ and $\sigma_{W}=0.05$, respectively, are not well correlated with the ranges of the original potentials. In particular, for the potential with the smaller range $\sigma_{W}=0.05$, the catch bond parameter is much larger. The reason for this might be that the fit value for the catch bond parameter corresponds effectively to an average over a whole range of the actual barrier position $y_{b}$, which is plotted in fig. $8 \mathrm{~b}$ ), in the relevant force range $0<f_{\text {ext }}<20$. In any case, we see that it might be difficult to reach the necessary small values of the catch bond parameter of the order of $x<0.02$ needed to observe shear-induced adsorption behavior (see fig. 3).

So far we obtained effective values for the catch bond parameter $x$ and the desorption energy barrier $E_{d}$. Our simulation results indicate that also the adsorption energy barrier height $E_{a}$ is a parameter that has to be finely tuned in order to observe shear-induced adsorption (see fig. $6 \mathrm{~b}$ ). Since escape rates do not include information about the adsorption energy barrier $E_{a}$, a second approach based on the monomer mobility is used next in order to estimate $E_{a}$. 


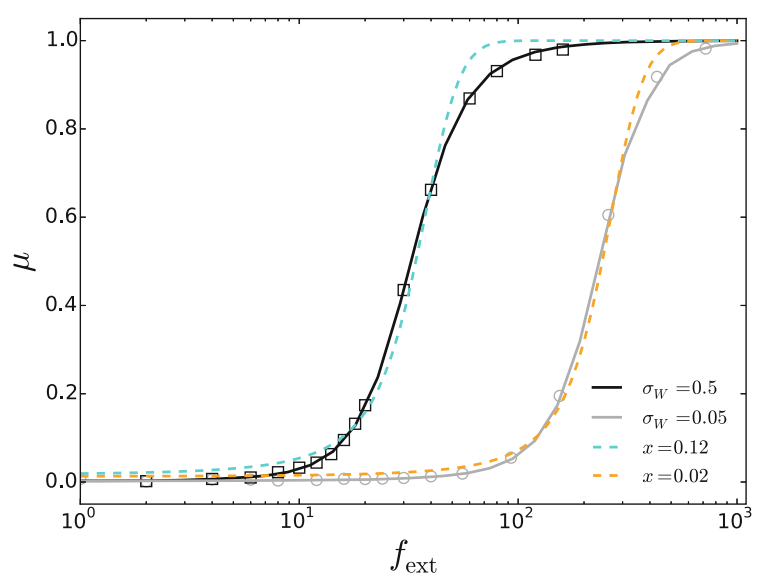

Fig. 9. Average particle mobility $\mu=V / f_{\text {ext }}$ in the onedimensional corrugated potential, eq. (8), as a function of pulling force $f_{\text {ext }}$. The average velocity $V$ is obtained from onedimensional BD simulations for $\sigma_{W}=0.5$ and $\varepsilon=10$ (black squares) as well as $\sigma_{W}=0.05$ and $\varepsilon=11.8$ (gray circles). Solid lines represent the solution of the corresponding Fokker-Planck equation, eq. (10). Colored dashed lines denote the fits according to a kinetic two-state model, eq. (11), with $\Delta E=4,4.3$ and $x=0.12,0.02$ for $\sigma_{W}=0.5,0.05$, respectively.

\subsection{Mobility in a one-dimensional corrugated potential}

In fig. 9 we plot the mobility $\mu=\tilde{\mu} / \mu_{0}=V / f_{\text {ext }}$, defined as the ratio of particle velocity $V$ and external force, of a particle in the one-dimensional corrugated potential eq. (8) as a function of the external force $f_{\text {ext }}$. Results from one-dimensional BD simulations, identical to the one used in sect. 4.1, indicated by open symbols, are in perfect agreement with the corresponding solution of the FokkerPlanck equation [28], indicated by solid lines and given by

$$
\begin{aligned}
\mu= & \frac{b\left(1-e^{-b f_{\text {ext }}}\right)}{f_{\text {ext }}}\left(\int_{0}^{b} e^{-U(y)} \mathrm{d} y \int_{0}^{b} e^{U\left(y^{\prime}\right)} \mathrm{d} y^{\prime}\right. \\
& \left.-\left(1-e^{-b f_{\text {ext }}}\right) \int_{0}^{b} \int_{0}^{y} e^{-U(y)+U\left(y^{\prime}\right)} \mathrm{d} y^{\prime} \mathrm{d} y\right)^{-1} .
\end{aligned}
$$

To extract desorption and adsorption rates from the data shown in fig. 9, we envision the particle motion as a sequence of adsorbed and desorbed states. We assume that the typical adsorption time is $t_{a}$, during which the particle is immobile, and that the typical desorption time is $t_{d}$, during which the particle moves over a distance $d$. The rescaled mobility follows as the ratio of the average velocity, $V=d /\left(t_{d}+t_{a}\right)$, and the velocity in the desorbed state, $V_{\mathrm{d}}=d / t_{d}$. Furthermore, the desorption time is related to the adsorption rate via $t_{d}=1 / k_{\mathrm{a}}$ and the adsorption time to the desorption rate via $t_{a}=1 / k_{\mathrm{d}}$. We obtain for the mobility

$$
\mu=\frac{V}{V_{\mathrm{d}}}=\frac{t_{d}}{t_{a}+t_{d}}=\frac{1}{1+k_{\mathrm{a}} / k_{\mathrm{d}}}=\frac{1}{1+e^{(\Delta E-x f)}},
$$

where in the last step we have used eqs. (5) and (6). Equation (11) thus allows to extract the catch bond parame- ter $x$ and the adsorption energy $\Delta E$ from the potentialbased model by fit of the mobility. We obtain the fit values $\Delta E=4$ and $x=0.12$ for the potential parameters $\sigma_{W}=0.5$ and $\varepsilon_{W}=10$, and $\Delta E=4.3$ and $x=0.02$ for $\sigma_{W}=0.05$ and $\varepsilon_{W}=11.8$, the corresponding fit functions are shown in fig. 9 by dashed lines.

We see that we obtain quite different fit values for the catch bond parameter $x$ based on the desorption rate in sect. 4.1 or based on the mobility in the present section. For the potential parameters $\sigma_{W}=0.05$ and $\varepsilon_{W}=11.8$ we obtain $x=0.2$ from the desorption rate and $x=0.02$ from the mobility. This shows that even a simple surfacemonomer potential landscape as in eq. (7) cannot be easily and unambiguously cast into a stochastic two-state model. This might be due to the fact that the desorption rate can be fitted to the two-state model reasonably only for small forces $f_{\text {ext }}<20$, as seen in fig. 8c), because the desorption barrier in a realistic potential landscape does not depend in a linear fashion on the applied force, as is assumed in our desorption rate expression eq. (6). In contrast, the mobility is fitted well by the two-state model in a much broader range of $f_{\text {ext }}$, as seen in fig. 9 , here the non-linear force dependence of the desorption barrier height seems to be less important. Alternatively, the reason for the ambiguous fitting results for $x$ could be that also the adsorption barrier exhibits a force dependence, which is neglected in our simple adsorption rate expression in eq. (5).

Extracting the desorption barrier height from the desorption rate we obtain for $\sigma_{W}=0.05$ and $\varepsilon_{W}=11.8$ the value $E_{d}=10.42$. This value is comparable to the range where we see shear-induced adsorption in fig. 6b). From a fit to the particle mobility we obtain for $\sigma_{W}=0.05$ and $\varepsilon_{W}=11.8$ the estimate $\Delta E=4.3$. This value is substantially larger than what is required for shear-induced adsorption, as seen in fig. 6a). The effective catch bond parameter we extract for $\sigma_{W}=0.05$ and $\varepsilon_{W}=11.8$ is either $x=0.2$ or $x=0.02$, depending on whether we use the desorption rate or the mobility for the fit. Disregarding the fact that it is not quite clear what the effective catch bond parameter actually is, it seems that even more highly corrugated potential landscapes for the surface-monomer binding would be needed in order to actually reach the effective catch bond parameter range $-0.02<x<0.02$ needed for shear-induced adsorption, as seen in fig. 3 .

In summary, if shear-induced globule adsorption is to be observed using a corrugated surface-monomer potential based on monomer-surface site pair potentials, the potential parameters should be chosen such as to mimic effective rate parameters in the range $E_{d}=9, \Delta E=0$ and $-0.02<x<0.02$. We argue that it should be possible to reach small effective catch bond parameters $x$ by choosing a very small surface interaction range $\sigma_{W}$, though this will make simulations quite inefficient because of the necessary small time steps. In order to achieve the necessary low $\Delta E$ value an additional adsorption barrier might have to be introduced in the potential-based model, such that the effective adsorption barrier $E_{a}$ becomes similar to $E_{d}$ and thereby $\Delta E=E_{d}-E_{a}$ approaches zero. 


\section{Summary and conclusion}

In the present study we investigate the adsorption of a polymeric globule in shear onto a surface with surfacemonomer bonds that obey stochastic two-state kinetics. We carefully determine the range of the surface-monomer bond parameters, i.e. the adsorption and desorption energy barriers $E_{a}$ and $E_{d}$ as well as the catch bond parameter $x$, for which shear-induced globule adsorption is obtained. As our main result, we find that in order to observe shear-induced adsorption, the catch bond parameter $x$ must be finely tuned in a narrow range $-0.02<x<0.02$, while the adsorption and desorption energy barriers must be quite high and set in a range of $5<E_{a}, E_{d}<12$. Interestingly, the energy barrier difference $\Delta E=E_{d}-E_{a}$, which corresponds to the adsorption energy, must be finely tuned in a narrow range of $-0.3<\Delta E<0.4$. In other words, the globule must be close to the equilibrium adsorption transition. This corresponds to very stringent conditions on the system parameters and means that biological systems must be finely adjusted in order for flow effects to enhance adsorption of globular structures on surfaces.

The physical picture [18] is that the polymer approaches the surface in a globular conformation in which desorbing lift forces are minimal. Since only few monomers are in the reaction range of the surface, the rather large adsorption energy barrier prevents globule adsorption at low shear. However, when a bond is formed and the shear flow is high enough, the chain is stretched and more bonds can form, given the bond lifetime of the initially formed bond is larger than the globule unfolding time. Our results show that even slip bonds, characterized by a catch bond parameter of the order of $x=0.01$, can give rise to shear-enhanced globule adsorption.

We show that the shear-induced adsorbed state is reversible with respect to switching off the shear flow, meaning that an adsorbed globule for suitably chosen parameters rapidly desorbs when the shear rate is suddenly set to zero. Similar reversibility has been seen previously in simulations of shear-induced polymer-colloid aggregate formation [29]. At the same time, large shear rates and large desorption energy barriers give rise to severe sampling problems which result in irreversibility effects in the simulations.

In the last part, we attempt a mapping of the kinetic two-state model parameters onto a potential-based binding model. The goal here is to understand the specificities of a surface-monomer pair potential that would be needed in order to lead to shear-induced adsorption of a globule. In a somewhat broader context, the question here is how catch bond behavior results from macromolecules that interact via conservative pair potentials. We perform the mapping using two scenarios, in the first scenario we calculate particle desorption rates, in the second scenario we calculate mobilities of a particle that is dragged over a onedimensional highly corrugated potential landscape. Catch bond parameters in the range of $x=0.02-0.4$ are obtained, while the adsorption energy difference $\Delta E$ turns out to be substantially larger than what is required for shear-enhanced adsorption. This suggests that it might be necessary to add an adsorption barrier into the surface monomer interaction potential. We conclude that it is not straightforward to design a model based on conservative surface-monomer pair potentials that would lead to shear-induced adsorption behavior. On the other hand, the shortcomings of our mapping also show that the description of surface-monomer interactions using a kinetic two-state model with only three parameters is incomplete and an additional parameter reflecting the forcedependence of the adsorption rate might be needed.

The interesting question remains of how catch bond behavior can be reproduced by models based on pair potentials. Clearly, the force dependence of the stability of a potential-based bond depends on the potential shape in a non-trivial fashion, and it is altogether not clear what a minimal model to obtain effective catch bond behavior is. It might be possible to induce the catch bond character of a bond by introducing saturation and shielding effects, which would involve the interplay of several binding sites.

The authors thank Alfredo Alexander-Katz for stimulating discussions and acknowledge financial support from the DFG Research Unit FOR 1543.

Open Access This is an open access article distributed under the terms of the Creative Commons Attribution License (http://creativecommons.org/licenses/by/4.0), which permits unrestricted use, distribution, and reproduction in any medium, provided the original work is properly cited.

\section{References}

1. M. Dembo, D.C. Torney, K. Saxman, D. Hammer, Proc. R. Soc. Lond., B, Biol. Sci. 234, 55 (1988) PMID: 2901109.

2. Evgeni V. Sokurenko, Viola Vogel, Wendy E. Thomas, Cell Host \& Microbe 4, 314 (2008).

3. Bryan T. Marshall, Mian Long, James W. Piper, Tadayuki Yago, Rodger P. McEver, Cheng Zhu, Nature 423, 190 (2003).

4. Tadayuki Yago, Jizhong Lou, Tao Wu, Jun Yang, Jonathan J. Miner, Leslie Coburn, Jose A. Lopez, Miguel A. Cruz, Jing-Fei Dong, Larry V. McIntire, Rodger P. McEver, Cheng Zhu, J. Clin. Invest. 118, 3195 (2008) PMID: 18725999; PMCID: PMC2518822.

5. W.E. Thomas, V. Vogel, E. Sokurenko, Annu. Rev. Biophys. 37, 399 (2008).

6. Jongseong Kim, Cheng-Zhong Zhang, Xiaohui Zhang, Timothy A. Springer, Nature 466, 992 (2010).

7. Sabyasachi Rakshit, Yunxiang Zhang, Kristine Manibog, Omer Shafraz, Sanjeevi Sivasankar, Proc. Natl. Acad. Sci. U.S.A. 109, 201208349 (2012) PMID: 23112161.

8. Jizhong Lou, Cheng Zhu, Biophys. J. 92, 1471 (2007).

9. Kristine Manibog, Hui Li, Sabyasachi Rakshit, Sanjeevi Sivasankar, Nat. Commun. 5, 3941 (2014)

10. Hsieh Chen, Alfredo Alexander-Katz, Biophys. J. 100, 174 (2011).

11. T.A. Springer, J. Thromb. Haemost. 9, 130 (2011).

12. S.W. Schneider, S. Nuschele, A. Wixforth, C. Gorzelanny, A. Alexander-Katz, R.R. Netz, M.F. Schneider, Proc. Natl. Acad. Sci. U.S.A. 104, 7899 (2007). 
13. Z.M. Ruggeri, J. Thromb. Haemost. 1, 1335 (2003).

14. M. Radtke, R. Netz, Eur. Phys. J. E 37, 20 (2014).

15. C. Sendner, R.R. Netz, EPL 81, 54006 (2008).

16. C.E. Sing, A. Alexander-Katz, EPL 95, 48001 (2011).

17. C.E. Sing, J.G. Selvidge, A. Alexander-Katz, Biophys. J. 105, 1475 (2013).

18. Alfredo Alexander-Katz, Macromolecules 140219083331009 (2014).

19. G. Bell, Science 200, 618 (1978).

20. C.B. Korn, U.S. Schwarz, Phys. Rev. E 77, 041904 (2008).

21. Hsieh Chen, Alfredo Alexander-Katz, Soft Matter 9, 10381 (2013).

22. Jens Rotne, Stephen Prager, J. Chem. Phys. 50, 4831 (1969).
23. J.R. Blake, Proc. Camb. Philos. Soc. 70, 303 (1971).

24. Y. von Hansen, M. Hinczewski, R.R. Netz, J. Chem. Phys. 134, 235102 (2011).

25. M. Doi, S.F. Edwards, The Theory of Polymer Dynamics, Vol. 73 (Oxford University Press, 1988).

26. T.R. Einert, C.E. Sing, A. Alexander-Katz, R.R. Netz, Eur. Phys. J. E 34, 130 (2011).

27. Peter Hänggi, Peter Talkner, Michal Borkovec, Rev. Mod. Phys. 62, 251 (1990).

28. A. Serr, C. Sendner, F. Müller, T.R. Einert, R.R. Netz, EPL 92, 38002 (2010).

29. H. Chen, M.A. Fallah, V. Huck, J.I. Angerer, A.J. Reininger, S.W. Schneider, M.F. Schneider, A. AlexanderKatz, Nat. Commun. 4, 1333 (2013). 